\title{
Schistosoma mansoni venom allergen-like protein 18 (SmVAL18) is a plasminogen-binding protein secreted during the early stages of mammalian-host infection
}

\author{
Rafaela S. Fernandes ${ }^{\mathrm{a}, \mathrm{b}}$, Luis G.V. Fernandes ${ }^{\mathrm{a}, \mathrm{b}}$, Andre S. de Godoy ${ }^{\mathrm{c}}$, Patrícia A. Miyasato ${ }^{\mathrm{d}}$, \\ Eliana Nakano $^{\mathrm{d}}$, Leonardo P. Farias ${ }^{\mathrm{e}, * *}$, Ana L.T.O. Nascimento ${ }^{\mathrm{a}}$, Luciana C.C. Leite ${ }^{\mathrm{a}, *}$ \\ a Laboratorio de Desenvolvimento de Vacinas, Instituto Butantan, Av. Vital Brasil, 1500, São Paulo, SP, Brazil \\ b Programa de Pós-Graduação Interunidades em Biotecnologia, Universidade de São Paulo, São Paulo, SP, Brazil \\ ${ }^{\mathrm{c}}$ Instituto de Física de São Carlos, Universidade de São Paulo, São Carlos, SP, Brazil \\ d Laboratório de Parasitologia, Instituto Butantan, Av. Vital Brasil, 1500, São Paulo, SP, Brazil \\ e IGM - Fundação Oswaldo Cruz-FIOCRUZ, Rua Waldemar Falcão, 121, 40296-710 Salvador, BA, Brazil
}

\section{A R T I C L E I N F O}

\section{Keywords:}

Schistosomiasis

SCP/TAPS

SmVAL18

Plasminogen

PLG-binding protein

\begin{abstract}
A B S T R A C T
Schistosomiasis is a neglected tropical disease caused by trematodes of the genus Schistosoma which have a complex life cycle characterized by an asexual multiplication phase in the snail intermediate host and a sexual reproduction phase in the mammalian definitive host. The initial steps of the human host infection involve the secretion of proteins contained in the acetabular glands of cercariae that promote parasite adhesion and proteolysis of the skin layers. Herein, we performed a functional analysis of SmVAL18, identified as one of the three SCP/TAPS proteins constituent of cercarial secretions. We evaluated the SmVAL18 binding to immobilized macromolecules of the extracellular matrix (ECM) and to plasma components. Recombinant protein, expressed in E. coli, was found to maintain an ordered secondary structure typical of the SCP/TAPS domain after purification. Expression of native SmVAL18 protein was verified to be restricted to cercariae and 3-h schistosomula stages; furthermore, the protein was observed in the corresponding secretions, confirming that SmVAL18 is secreted during the first $3 \mathrm{~h}$ of in vitro culture. rSmVAL18 was able to interact specifically with plasminogen (PLG) and enhance its conversion into plasmin in the presence of the urokinase-type plasminogen activator (uPA). Protein homology modelling suggested that the PLG-rSmVAL18 interaction was mediated by lysine residues of the protein. This was supported by in vitro data using the lysine analogue, 6-aminocaproic acid (ACA), which abolished the interaction. Finally, our results showed that both cercariae and 3-h schistosomula, as well as their corresponding secretions, exhibited the capacity to bind PLG and enhance its conversion into plasmin in vitro in the same way as observed for the recombinant protein. In conclusion, our findings show that SmVAL18 is a novel PLG-binding protein secreted during the early stages of the mammalian-host infection.
\end{abstract}

\section{Introduction}

Schistosomiasis is a neglected tropical disease that affects approximately 230 million people worldwide with close to 800 million living at risk of infection. Schistosoma mansoni, one of the three major causing agents of the disease, has a complex life cycle, which involves an asexual multiplication phase in the snail intermediate host and a sexual reproduction phase in the mammalian definitive host [1,2]. Adult worms living within the mesenteric veins are constantly producing eggs that either become trapped in the tissues inducing an inflammatory response or are released into the environment by the feces. The eggs reach the freshwater supply and hatch releasing the ciliated miracidia, the larval stage that infects a suitable snail host. In the intermediate host, the parasite undergoes asexual replication through mother and daughter sporocysts within which germballs develop culminating with the shedding of thousands of cercariae into the water [2,3].

The human infection is initiated by the contact of cercariae, the infective larval stage, with the lipids on the surface of the skin

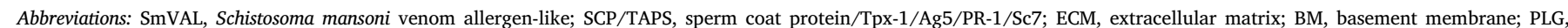
plasminogen; uPA, urokinase-type plasminogen activator

* Corresponding author at: Centro de Biotecnologia, Instituto Butantan, Av. Vital Brasil, 1500, 05503-900 São Paulo, SP, Brazil.

** Corresponding author.

E-mail addresses: leonardo.farias@bahia.fiocruz.br (L.P. Farias), luciana.leite@butantan.gov.br (L.C.C. Leite).
} 
triggering the mechanical entry into the cornified superficial epidermal layer. However, further penetration demands the degradation of both the basement membrane (BM) and the extracellular matrix (ECM) of the dermis [4]. All invasion processes are assisted by the secretion of proteins contained in the acetabular glands of cercariae that promote parasite adhesion to the skin and proteolysis of the intracellular bridges between epidermal/dermal cells [4-8].

The major components of the cercarial secretions were identified as isoforms of the serine protease cercarial elastase, capable of digesting elastin, and the metalloprotease SmPepM8, likely to play a role in the degradation of skin BM and ECM [4,5]. The minor components released, on the other hand, were all proposed to be immunomodulators. Three novel proteins containing the SCP (Sperm Coat Protein) domain, that have since been classified as members of the SmVAL family (Schistosoma mansoni Venom Allergen Like-Protein), SmVAL4, 10 and 18 , were identified representing $\sim 3 \%$ of the released content $[5,9,10]$.

Of the total 29 SmVAL genes identified to date (SmVAL1-29), 24 encode proteins with a secretion signal peptide (group 1 ), while only 5 encode intracellular proteins (group 2). Some of those were identified to have a stage specific expression with up-regulation in germballs (the precursor stage of mature cercariae - SmVAL4, 18, 19, 20 and 24), cercariae (SmVAL1, 2, 16, 17 and 21) and 3-day schistosomula (SmVAL7 and 13), raising questions on the involvement of SmVAL proteins with the mammalian host invasion [9,11-13].

In the last four years, we have focused on studying the tissue expression patterns of SmVAL genes as a way to infer possible functions to the respective proteins. We have previously described the localization of group 1 SmVAL7 and group 2 SmVAL6 transcripts in the posterior esophageal gland and in the suckers (oral and ventral) of adult worms, respectively [14]. Recently, we identified SmVAL13 transcript in the anterior esophageal gland of adult worms and localized SmVAL1, 4, 10, 18, 19 and 24 in the acetabular glands of germballs ([12] and Farias, personal communication). Furthermore, the expression profiles of SmVAL21, 22 and 25 suggest the same localization (Farias, personal communication). Thus, the high number of different SmVALs putatively expressed in the acetabular glands draws attention to a hotspot for this gene family expression in this structure.

To date, only two group 1 SmVAL proteins were associated with specific functions. SmVAL9, secreted during miracidia to sporocyst transformation in vitro, was found to stimulate transcription of genes involved in host ECM remodeling [13]. Native SmVAL4 was identified among the proteins secreted in the tunnels formed by destruction of epidermal cells by cercariae, during the first two hours of penetration of human skin [15]. Later, the protein was found to have the ability to bind lipids in vitro and complement the sterol export phenotype of yeasts in vivo $[15,16]$. So far, with the exception of these studies, no functional data have been associated to any other SmVALs.

Aiming to shed light on the role of other SmVALs secreted during the first hours of the mammalian-host invasion, herein we evaluated the SmVAL18 binding to immobilized macromolecules of the extracellular matrix (ECM), the tissue barrier that migratory larvae must overcome in order to reach a blood vessel, and to plasma components that could impair parasite migration. The data presented demonstrate the ability of SmVAL18 to bind plasminogen and increase its conversion into plasmin in the presence of urokinase-type plasminogen activator (uPA). Data are discussed in the light of the predicted functions of acetabular secretions in the parasite penetration process.

\section{Materials and methods}

\subsection{Ethics statement}

All procedures involving animals were carried out in compliance with the Brazilian legislation (decree no. 11790/2008). All animals were handled in strict accordance with good animal practices, and the study protocol received approval by the Institutional Review Board for
Animal Research of the Butantan Institute (CEUAIB, São Paulo, Brazil) under license number 799/11.

\subsection{Parasites}

Schistosoma mansoni (BH strain) cercariae were first obtained by exposure of infected Biomphalaria glabrata snails to bright light and schistosomula were obtained by the mechanical transformation of cercariae followed by in vitro cultivation for $3 \mathrm{~h}$ and 3, 5 and 7 days as previously described [12].

\subsection{Cloning, expression and purification of recombinant SmVAL18}

After several unsuccessful attempts to obtain the full-length of SmVAL18 cDNA, a fragment corresponding to $84 \%$ of the mature protein sequence (from Pro55 to Tyr194), kindly provided by Dr. Iain Chalmers (Aberystwyth University), was used in the production of the recombinant protein. The cDNA sequence was cloned into PGEM $^{\circledR}$-T Easy vector (Promega), and then amplified by conventional PCR using the following primers: forward (F) $5^{\prime}$ TACGGATCCCCAAAACAACCTC CAGCA 3'; reverse (R) 5' TACGAATTCTTAATATTCTGCATCATCAAC 3'. This fragment was then subcloned into pAE vector [17] at the EcoRI and BamHI restriction sites for expression in E. coli. Plasmid pAESmVAL18 containing the confirmed DNA sequence was used to transform $E$. coli BL21 (DE3). For protein expression, a pre-inoculum of $3 \mathrm{~mL}$ was first grown overnight and inoculated into $300 \mathrm{~mL}$ of LB (Lysogeny broth) medium containing $50 \mu \mathrm{g} / \mathrm{mL}$ of ampicillin. The culture was incubated under continuous shaking (200 RPM) at $37^{\circ} \mathrm{C}$ to reach an optical density of $0.6-0.8$ at $600 \mathrm{~nm}$ (O.D. ${ }_{600}$ ). Protein expression was induced for $20 \mathrm{~h}$ at $18{ }^{\circ} \mathrm{C}$ with $1.0 \mathrm{mM}$ IPTG (isopropyl- $\beta$-D-1-galactopyranoside) under constant agitation.

Cells were harvested from the medium by centrifugation, resuspended in lysis buffer ( $50 \mathrm{mM}$ Tris $\mathrm{pH} 8.8 ; 300 \mathrm{mM} \mathrm{NaCl}$ ) and lysed by sonication $(60 \mathrm{~Hz}, 1.0 \mathrm{~s}$ pulse, $10 \mathrm{~min})$ in an ice bath. Following separation of the soluble and insoluble (inclusion bodies) fractions by centrifugation, rSmVAL18 was purified from the soluble fraction by nickel-affinity chromatography using a $5 \mathrm{~mL}$ His-Trap ${ }^{\mathrm{TM}} \mathrm{HP}$ column (GE Healthcare), then dialyzed in PBS (phosphate buffered-saline) at $4{ }^{\circ} \mathrm{C}$ for $48 \mathrm{~h}$. Protein purity was verified by $15 \%$ SDS-PAGE and the final rSmVAL18 concentration was determined by Lowry's method (DC Protein Assay - Bio-Rad) using bovine serum albumin as a standard.

\subsection{Circular dichroism spectroscopy}

Circular dichroism (CD) spectroscopy measurements were performed at $20^{\circ} \mathrm{C}$ in a Jasco J-810 Spectropolarimeter (Japan Spectroscopic) equipped with a Peltier unit for temperature control. The far-UV CD spectrum was acquired using a $1.0 \mathrm{~mm}$ path length cell at $0.5 \mathrm{~nm}$ intervals along wavelengths ranging from 185 to $260 \mathrm{~nm}$. Each scan was recorded as the average of five scans, and then subtracted from the average blank spectra. Protein concentration was maintained at $10 \mu \mathrm{M}$ in $10 \mathrm{mM}$ sodium phosphate buffer $\mathrm{pH}$ 8.0.

\subsection{Polyclonal antibody production}

BALB/c mice were subcutaneously immunized with $15 \mu \mathrm{g}$ of recombinant protein formulated with aluminum hydroxide at a 1:10 ratio (protein: adjuvant). Each animal received a total of three doses of this formulation at 14-day intervals. Retro-orbital bleedings were performed prior to each immunization and a final bleeding was conducted two weeks after the last immunization. Antibody titers were assessed by ELISA.

\subsection{Protein expression throughout larval stages in culture}

Total protein extracts of cercariae, as well as 3-h, 3-, 5- and 7-day 
schistosomula, in addition to their released proteins (denominated Cerc RP, 3-h, 3-, 5- and 7-day RP), were obtained as previously described [12]. Total parasite extracts were quantified using Lowry's method (DC Protein Assay - Bio-Rad). Purified rSmVAL18 (15 and $50 \mathrm{ng}$ ), total parasite extracts $(10 \mu \mathrm{g})$ and released proteins $(20 \mu \mathrm{L}$ of a $100 \mathrm{x}$ concentrated supernatant from a $7.5 \mathrm{~mL}$ culture containing 70,000 parasites) were submitted to $15 \%$ SDS-PAGE. Proteins were electroblotted onto a PVDF membrane (GE Healthcare) and Western blotting was performed as previously described [12], using anti-rSmVAL18 (1:2000) polyclonal antibodies.

\subsection{ECM and plasma components}

Macromolecules, including thrombin and the control proteins fetuin and gelatin, were purchased from Sigma-Aldrich. Laminin-1 and collagen type IV were derived from the basement membrane of Engelbreth-Holm-Swarm mouse sarcoma; cellular fibronectin was derived from human foreskin fibroblasts; plasma fibronectin, human complement serum and fibrinogen were isolated from human plasma, while collagen type I was isolated from rat tail. Native plasminogen, purified from human plasma, and human factor $\mathrm{H}$ were purchased from EMD Chemicals, Inc. C4 bp was obtained from Complement Technology, Inc.

\subsection{Binding of rSmVAL18 to the ECM and plasma components}

Protein attachment to individual extracellular matrix and plasma components was analyzed as previously described [18]. ELISA plates (Corning) were coated with $1 \mu \mathrm{g}$ of the components and the negative controls: 1\% BSA (nonglycosylated attachment-negative control protein), $1 \mu \mathrm{g}$ of fetuin (highly glycosylated attachment-negative control protein) and $1 \%$ gelatin diluted in $100 \mu \mathrm{L}$ of PBS for $16 \mathrm{~h}$ at $4{ }^{\circ} \mathrm{C}$. The plates were then blocked with PBS containing 0.05\% Tween-20 (PBST) $1 \%$ gelatin for $2 \mathrm{~h}$ at $37^{\circ} \mathrm{C}$. After blockage, $500 \mathrm{ng}$ of rSmVAL18 was added to each well in $100 \mu \mathrm{L}$ of PBS $1 \%$ gelatin, then allowed to attach to the different substrates for $2 \mathrm{~h}$ at $37^{\circ} \mathrm{C}$. After three washes with PBST, the primary antibody (anti-rSmVAL18) was added to each well in a 1:4000 dilution of $100 \mu \mathrm{L}$ of PBS. Incubation proceeded for $1 \mathrm{~h}$ at $37^{\circ} \mathrm{C}$ and, after three washes with PBST, a 1:5000 dilution of $100 \mu \mathrm{L}$ of HRP-conjugated goat anti-mouse IgG (Southern Biotech) in PBS was added to each well, followed by incubation for $1 \mathrm{~h}$ at $37^{\circ} \mathrm{C}$. The plates were washed six times and $o$-phenylenediamine $(1.0 \mathrm{mg} / \mathrm{mL})$ in citratephosphate buffer ( $\mathrm{pH} 5.0$ ) plus $1 \mu \mathrm{L} / \mathrm{mL} \mathrm{H}_{2} \mathrm{O}_{2}$ was added $(100 \mu \mathrm{L}$ per well). Reactions were allowed to proceed for $5 \mathrm{~min}$ and then interrupted by the addition of $50 \mu \mathrm{L}$ of $\mathrm{H}_{2} \mathrm{SO}_{4} 4 \mathrm{~N}$. Finally, plates were read at $492 \mathrm{~nm}$ in a microplate reader (Epoch Microplate Spectrophotometer - BioTek).

\section{9. $K_{D}$ values for the binding of rSmVAL18 to thrombin and PLG}

For the determination of the dose-dependent attachment of rSmVAL18 to thrombin and PLG, increasing concentrations of the recombinant protein varying from 0 to $0.5 \mu \mathrm{M}$ in PBS was used. The assessment of protein binding was performed as described above in Section 2.8 (Binding of rSmVAL18 to the ECM and plasma components). Readings were used to calculate the equilibrium dissociation constant $\left(K_{D}\right)$, according to a previously described method [19], based on the equation: $A=A_{M A X}$ [protein] $/\left(K_{D}+\right.$ [protein]), where $A$ is the absorbance at a given protein concentration; $A_{M A X}$ is the maximum absorbance for the ELISA plate reader (equilibrium); [protein] is the protein concentration and $K_{D}$ is the equilibrium dissociation constant for a given absorbance at a given protein concentration (ELISA data point).

\subsection{Characterization of rSmVAL18 binding to plasminogen}

The binding of the recombinant protein to plasminogen (PLG) was characterized using a previously proposed protocol [20]. Briefly, a 96well plate was coated with $1 \mu \mathrm{g}$ of rSmVAL18 or $1 \%$ BSA (negative control) in $100 \mu \mathrm{L}$ of PBS per well for $16 \mathrm{~h}$ at $4{ }^{\circ} \mathrm{C}$, then blocked for $2 \mathrm{~h}$ at $37^{\circ} \mathrm{C}$. After blockage, $1 \mu \mathrm{g}$ of PLG was added to each well to interact with immobilized rSmVAL18 for $2 \mathrm{~h}$ at $37^{\circ} \mathrm{C}$. Next, plates were washed three times in PBST and a $100 \mu \mathrm{L}$ final volume of PBS containing $4 \mathrm{ng}$ of urokinase-type plasminogen activator (uPA - Sigma-Aldrich) and $0.4 \mathrm{mM}$ of plasmin chromogenic substrate (D-valyl-leucyl-lysine-p-nitroanilide dihydrochloride - Sigma-Aldrich) were added to wells. Controls lacked one of the reaction components (PLG, uPA or substrate). After $16 \mathrm{~h}$ of incubation at $37^{\circ} \mathrm{C}$, substrate degradation was assessed by O.D. measurements in a microplate reader at a wavelength of $405 \mathrm{~nm}$ [20].

To evaluate the role of lysine residues in the binding of rSmVAL18 to PLG, the recombinant protein was added to PLG-coated wells, together with 6-aminocaproic acid (ACA - Sigma Aldrich), a lysine analog, at concentrations of $0.2,2.0$ or $20 \mu \mathrm{M}$. The detection of bound protein was determined as described above.

\subsection{Molecular modeling of rSmVAL18}

Homology models of rSmVAL18 were generated using x-ray structural information from SmVAL4 (PDB id 4P27, 47\% sequence identity) via MODELLER software [21] using a HHpred server [22]. The model was validated with the Molprobity program [23], and the PyMOL (Molecular Graphics System, Version 1.8 Schrödinger, LLC) was used for structural analysis and to construct figures.

\subsection{PLG activation by live parasites}

The evaluation of PLG activation was based on the amylolytic activity of plasmin generated by live worms or, since SmVAL18 is secreted, by the native protein present in parasite secretions, using an adapted protocol [24]. Cercariae were collected from infected snails, then washed and incubated in PBS. Three-hour old schistosomula were obtained from cercariae mechanically transformed in PBS as previously described [12]. PBS containing the proteins secreted during transformation (denominated Cercariae Released Protein - Cerc RP) was stored at $4{ }^{\circ} \mathrm{C}$ to avoid protein degradation. After a $3 \mathrm{~h}$ incubation at $37^{\circ} \mathrm{C}$ under $5 \% \mathrm{CO}_{2}$, parasites were centrifuged at $2000 \times g$ for $3 \mathrm{~min}$ followed by the collection of both 3-h schistosomula and the secreted proteins (denominated 3-h Released Protein - 3-h RP). Cercariae and 3$\mathrm{h}$ schistosomula (approximately 1000 per experimental replicate) were incubated in $100 \mu \mathrm{L}$ of PBS with $1 \mu \mathrm{g}$ of PLG in a $1.5 \mathrm{~mL}$ tube for $2 \mathrm{~h}$ at $37^{\circ} \mathrm{C}$. Parasites were then washed three times in PBST, followed by the addition of a final volume of $100 \mu \mathrm{L}$ of PBS containing $4 \mathrm{ng}$ of uPA (Sigma-Aldrich) along with $0.4 \mathrm{mM}$ of plasmin chromogenic substrate (Sigma-Aldrich). Controls lacked one of the reaction components (PLG, uPA or substrate). Substrate degradation was assessed after a $16 \mathrm{~h}$ incubation at $37^{\circ} \mathrm{C}$ using O.D. measurements obtained in a microplate reader at a wavelength of $405 \mathrm{~nm}$. Cerc RP and 3-h RP were immobilized on ELISA plates and incubated with PLG for plasmin activity measurement, as described above.

\subsection{Statistical analysis}

All experiments involving interactions between the recombinant proteins, live parasites or released proteins and ECM/plasma components were performed in triplicate. Obtained absorbance values were compared to controls (including the negative control 1\% BSA) using the Student's $T$-test. A p value $<0.05$ was considered statistically significant. 
A

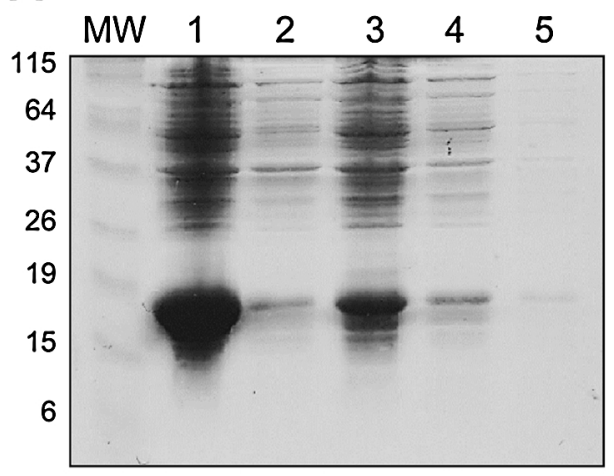

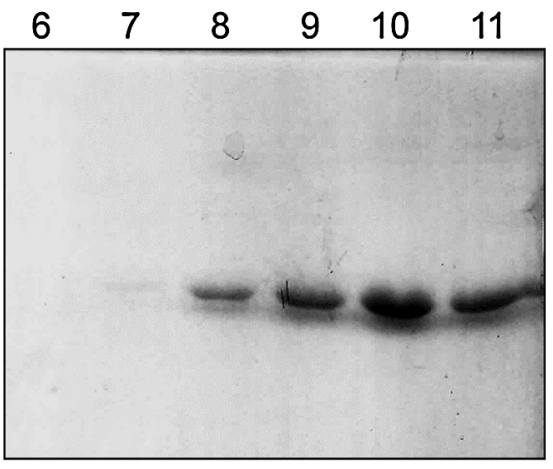

Fig. 1. Expression, purification and secondary structure analysis of recombinant SmVAL18. (A) SDSPAGE (15\%) analysis of fractions of the recombinant protein purification through $\mathrm{Ni}^{+2}$-charged column chromatography. Lane 1 - Supernatant after lysis of $E$. coli BL21 (DE3) expressing rSmVAL18; lanes 2-5 flow-through; 6-11 - fractions of rSmVAL18 eluted by linear gradient of imidazole $(20-500 \mathrm{mM})$. (B) Circular dichroism spectra of rSmVAL18 (an average of five measurements).

B

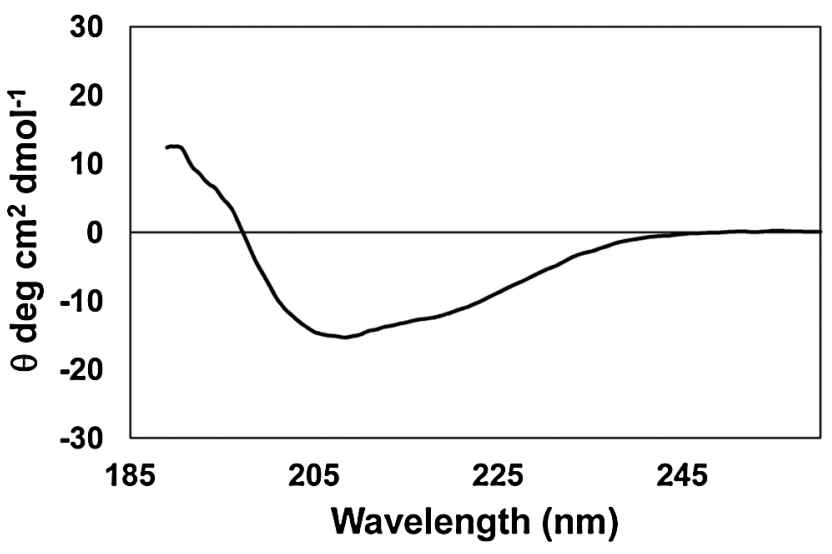

\section{Results}

\subsection{Expression and purification of recombinant SmVAL18}

A SmVAL18 cDNA fragment was cloned into 6His-pAE vector [17] for expression in E. coli BL21 (DE3). The protein was purified from the soluble bacterial fraction and a total of $5 \mathrm{mg} / \mathrm{L}$ of cell culture of rSmVAL18 was obtained after dialysis in PBS. Purified rSmVAL18 migrated as a single band of approximately $16 \mathrm{kDa}$ (predicted MW of $16.05 \mathrm{kDa}$ ) on $15 \%$ SDS-PAGE (Fig. 1A).

\section{2. rSmVAL18 secondary structure analysis}

Circular dichroism spectra of rSmVAL18 displayed a positive peak at $193 \mathrm{~nm}$ and two discrete negative peaks at approximately $208 \mathrm{~nm}$ and $222 \mathrm{~nm}$, indicating the presence of abundant $\alpha$ helices [25]. This ordered secondary structure strongly resembles that previously described for recombinant SmVAL4 produced in Pichia Pastoris [26], which consists of a three-layer alfa-beta-alfa sandwich, typical of the SCP/TAPS domain (Fig. 1B). The proportions of $\alpha$-helix and $\beta$-sheets were not estimated.

\subsection{Protein expression throughout the transition larval stages of cercariae to 7-day schistosomula in vitro}

Total protein extracts prepared from parasite stages cultivated in vitro for seven days (Cercariae to 7-day schistosomula) and also the corresponding proteins released in culture medium (Cerc RP to 7-day RP) were separated by SDS-PAGE and analyzed by immunoblotting using mouse polyclonal anti-rSmVAL18 antibodies. The obtained protein expression profile was very specific and restricted to cercariae and 3-h schistosomula stages (Fig. 2A). Moreover, native SmVAL18 was also
A

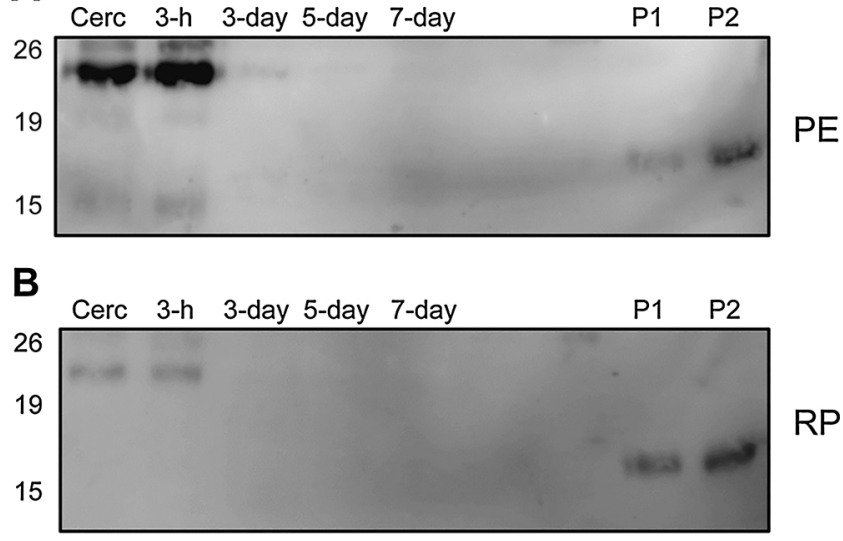

Fig. 2. Identification of native SmVAL18 in total protein extracts and secretions of larval stages cultured in vitro. Polyclonal anti-rSmVAL18 was used to identify native SmVAL18 in total parasite extracts (PE) and secretions containing released proteins (RP) of Cercariae (Cerc), 3-h schistosomula (3-h); day 3 schistosomula (3-day); day 5 schistosomula (5-day) and day 7 schistosomula (7-day); P1 and P2 - Positive controls, recombinant SmVAL18, 15 and $50 \mathrm{ng}$, respectively. For PE, $10 \mu \mathrm{g}$ of protein extract was applied in each lane (A). Secretions derived from $\sim 70.000$ cultured schistosomula $(20 \mu \mathrm{L}$ of a $100 \times$ concentrated supernatant from a $7.5 \mathrm{~mL}$ culture) were used in (B). Molecular weight is marked on the left side of the images $(\mathrm{kDa})$.

present in the secretions corresponding to those stages, denominated Cerc RP and 3-h RP (Fig. 2B). The native protein is visible as a single band of approximately $24 \mathrm{kDa}$. The differences observed between the molecular weights of native SmVAL18 $(\sim 24 \mathrm{kDa})$ and the recombinant protein $(\sim 16 \mathrm{kDa})$ are due to the fact that the rSmVAL18 sequence represents $84 \%$ of the native protein, and also the result of $\mathrm{N}$ - and $\mathrm{O}-$ glycan structures in the native protein, which have been previously 

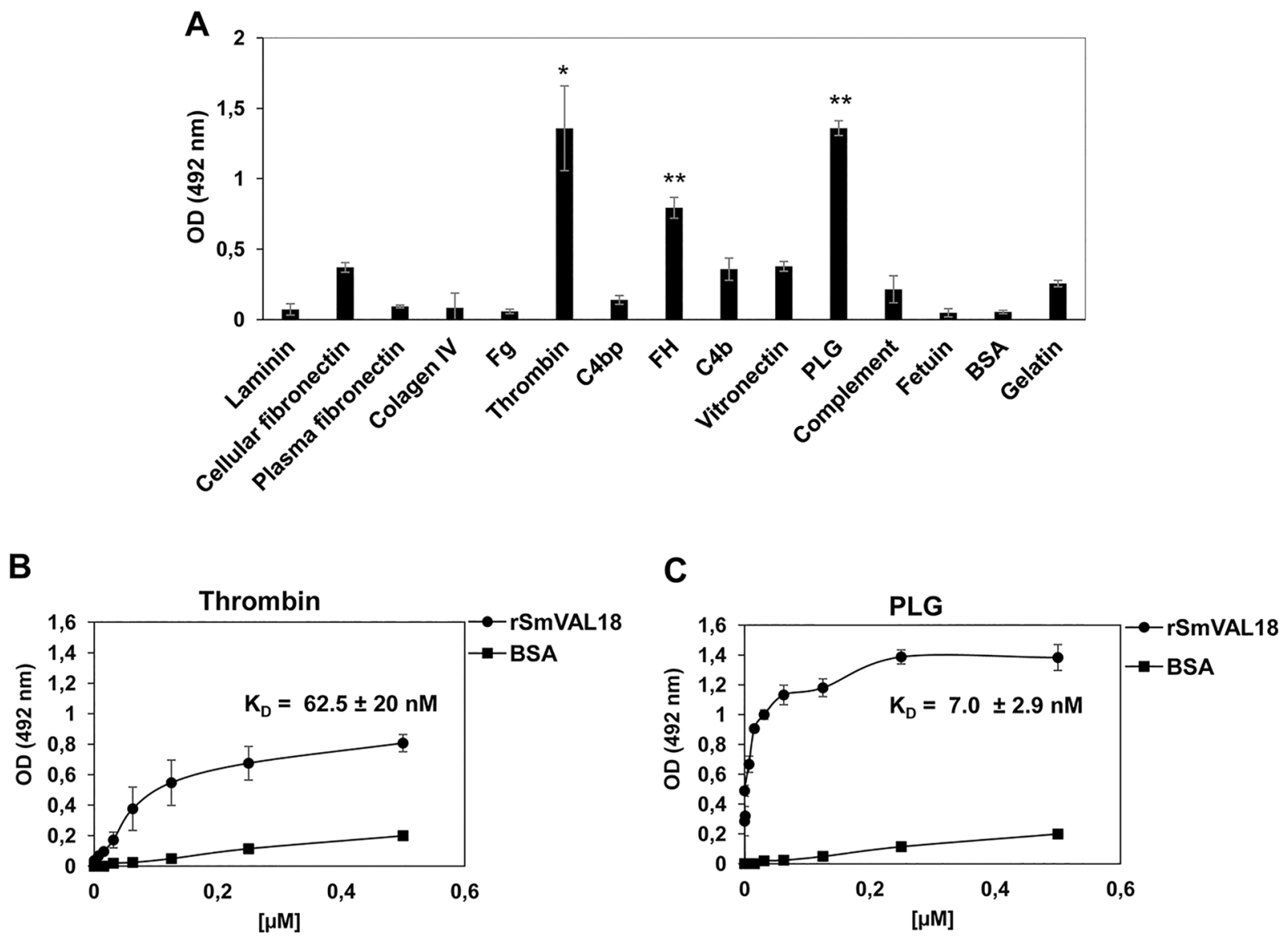

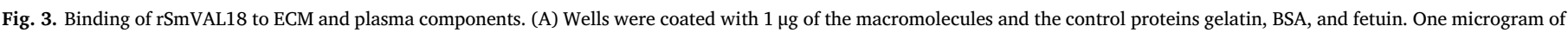

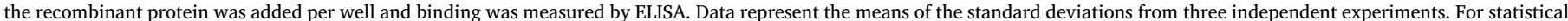

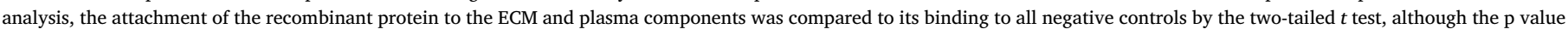

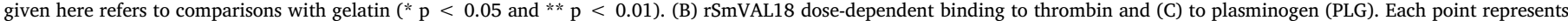

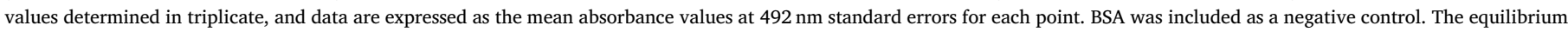

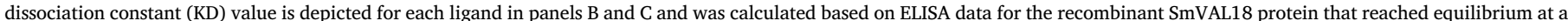
given concentration.

described $[5,10]$.

\subsection{Binding of rSmVAL18 to ECM components}

The binding of the recombinant protein to ECM and plasma components was evaluated by ELISA. Target molecules and negative controls (BSA, fetuin and gelatin) were immobilized on a 96-well plate and the protein attachment was analyzed as previously described [18]. As shown in Fig. 3A, rSmVAL18 exhibited statistically significant binding to thrombin, factor $\mathrm{H}(\mathrm{FH})$ and PLG.

\section{5. $K_{D}$ values of $r S m V A L 18$ binding to thrombin and $P L G$}

The interaction of rSmVAL18 with thrombin, FH and PLG was also assessed on a quantitative basis by constant maintenance of component concentrations while varying the protein concentration. Although the protein showed a statistically significant attachment to $\mathrm{FH}$, the obtained $K_{D}$ values were not significantly different from those of the control BSA (data not shown). A saturation level of binding was achieved at a protein concentration of approximately $0.5 \mu \mathrm{M}$ for thrombin and $0.25 \mu \mathrm{M}$ for PLG with corresponding $K_{D}$ values of $62.5 \pm 20 \mathrm{nM}$ and $7.0 \pm 2.9 \mathrm{nM}$, respectively (Fig. 3B and C).

\subsection{Plasminogen activation in vitro}

To investigate whether rSmVAL18-bound PLG could be converted into its active form, plasmin, a 96-well plate was coated with the recombinant protein and then incubated with PLG. After the addition of uPA and plasmin chromogenic substrate, the reaction was allowed to proceed for $16 \mathrm{~h}$ at $37^{\circ} \mathrm{C}$. As shown in Fig. 4A, the plasminogen bound to the recombinant protein was capable of conversion into plasmin, as indirectly demonstrated by specific proteolytic activity $(\mathrm{p}<0.05)$. Control reactions lacking one of the components of this system showed no significant enzymatic activity. The participation of lysine residues in recombinant protein binding was evaluated by adding ACA to the assay mixture. The binding of rSmVAL18 to PLG decreased with increasing concentrations of ACA, and was almost completely inhibited when $20 \mu \mathrm{M}$ of ACA was added to the reaction mixture (Fig. 4B), strongly suggesting the participation of lysine residues in rSmVAL18-PLG interaction.

\section{7. rSmVAL18 homology modelling}

Molecular modelling to assess this protein's structural features revealed that, similarly to the x-ray structure of rSmVAL4, the homology model of rSmVAL18 folds as a $\alpha-\beta-\alpha$ sandwich [27], in which a three anti-parallel $\beta$-sheet motif is situated between three $\alpha$-helices (Fig. 5). The model of rSmVAL18 shows 13 lysine residues positioned along the structure. A closer analysis reveals that lysines 7, 11, 16, 59, 69, 79, 102,121 and 126 are positioned on the molecular surface of this model (Fig. 5), thus forming the solvation layer of the protein. 


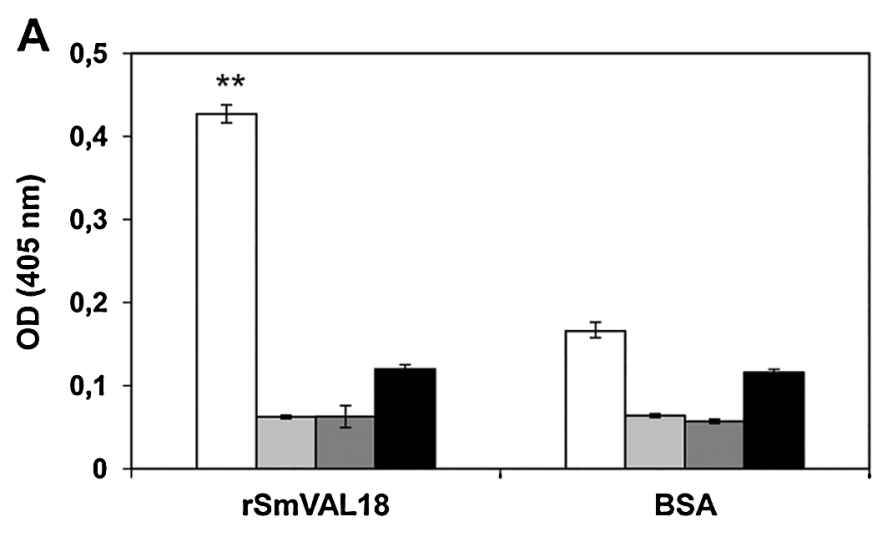

B

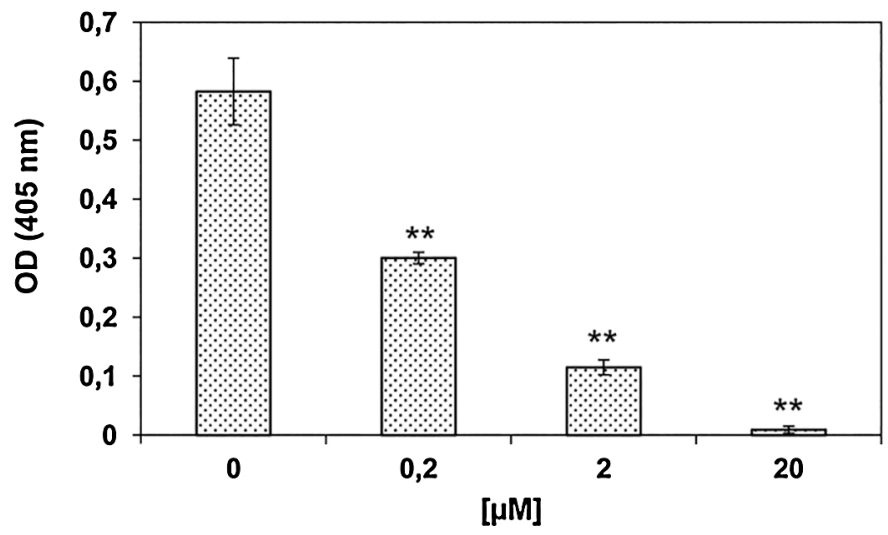

3.8. Plasminogen-activation by live parasites and secreted proteins

The native protein expression found herein was associated with both cercariae and 3-h schistosomula, and also identified in their secretions. Accordingly, the ability of plasminogen to bind to cercariae and 3-h schistosomula, as well as their corresponding released proteins, in addition to plasmin activation in vitro, were analyzed.

Plasminogen bound to both cercariae and 3-h schistosomula was activated into plasmin in the presence of uPA (Fig. 6A). This same result was observed in cercariae and 3-h schistosomula RPs (Fig. 6B). $\square P L G+U P A+S$

$\square$ PLG+UPA

$\square P L G+S$

UPA+S

QACA
Fig. 4. Plasmin generation by PLG bound to recombinant protein. The conversion of PLG in active plasmin was measured indirectly by the cleavage of the specific plasmin substrate assessed by a modified ELISA. (A) The immobilized recombinant SmVAL18 received the following treatment: PLG, UPA, and the specific plasmin substrate $S$ $(\mathrm{PLG}+\mathrm{uPA}+\mathrm{S})$ or controls lacking one of the three components $(\mathrm{PLG}+\mathrm{uPA}$; PLG $+\mathrm{S}$; $\mathrm{uPA}+\mathrm{S})$. BSA was used as negative control. Bars represent the mean absorbance values at $405 \mathrm{~nm}$, as a measure of the relative substrate cleavage. Standard deviations are from three replicates for each experimental group and are representative of three independent experiments. Statistically significant difference in comparison to BSA is shown ( ${ }^{* *} \mathrm{p}<0.01$ ). (B) Binding of rSmVAL18 to PLG was carried out in presence of the lysine analogue 6-aminocaproic acid (ACA). For statistical analysis, the attachment of the recombinant protein in the presence of ACA was compared to its binding to PLG without ACA (no inhibition at $0 \mu \mathrm{M}$ ) by the two-tailed $t$ test $(* *$ $\mathrm{p}<0.01)$.

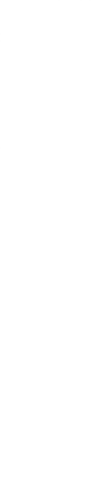

\section{Discussion}

The present study attempted to functionally characterize SmVAL18, which was previously identified as one of the three SCP/TAPS proteins present in cercarial secretions $[5,10]$. This gene was described as upregulated in the germball stage [28] and its transcript was localized within the acetabular glands (Farias, personal communication), reinforcing the hypothesis that this protein may play a role in the early stages of mammalian-host infection.

The recombinant protein expressed in $E$. coli was purified from the soluble fraction of this bacteria and showed an ordered secondary

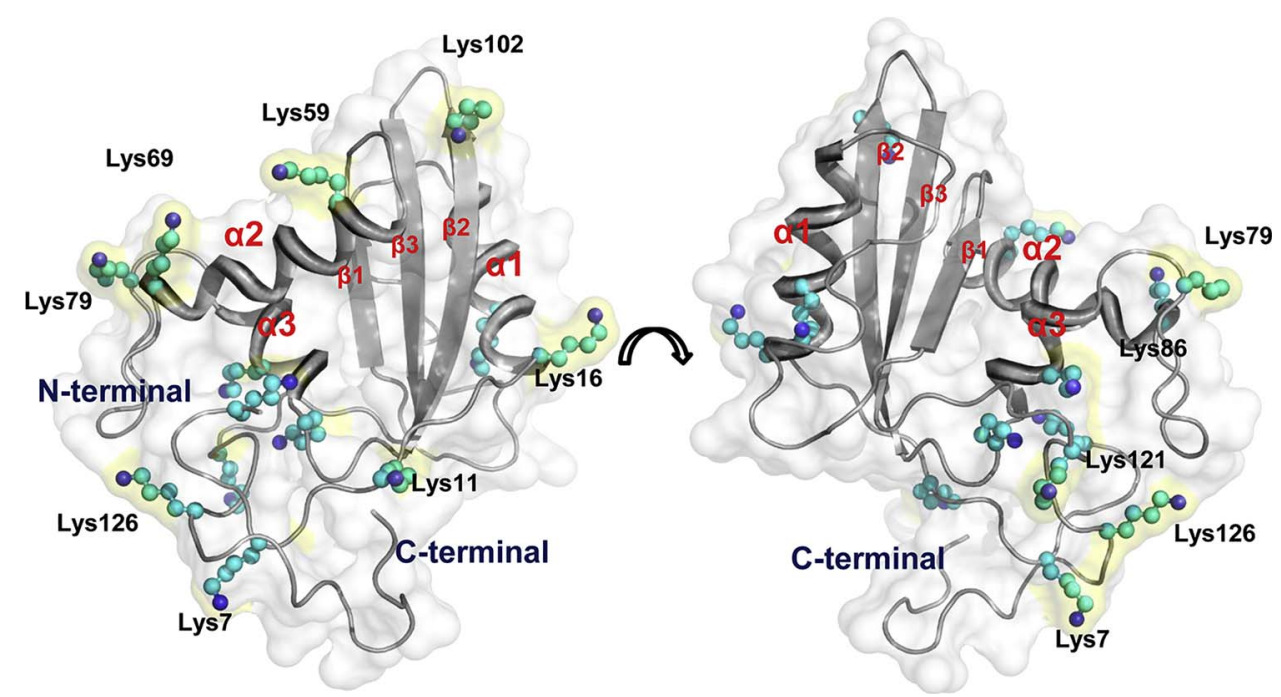

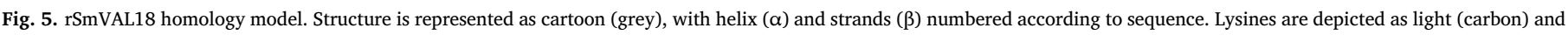
dark (nitrogen) blue spheres. (For interpretation of the references to colour in this figure legend, the reader is referred to the web version of this article.) 
A

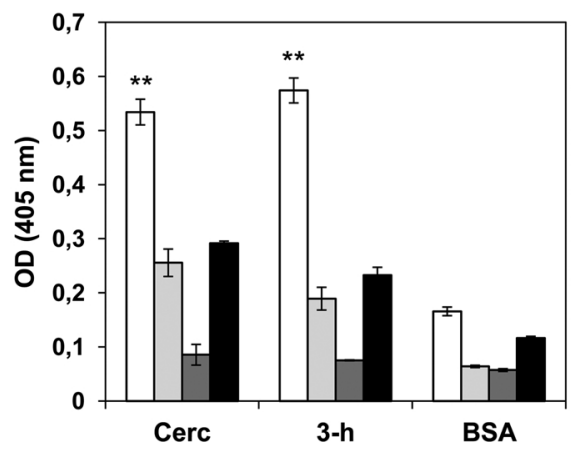

B

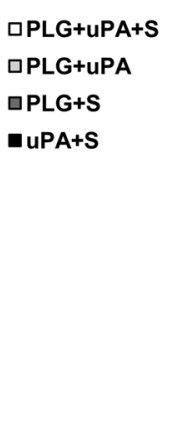

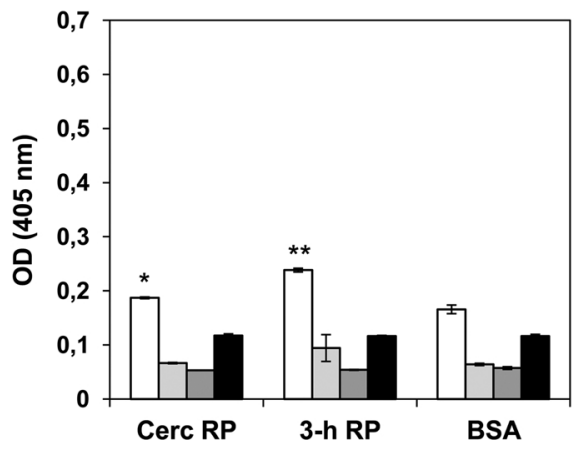

口PLG+uPA+S

$\square P L G+U P A$

$\square P L G+S$

uPA+S

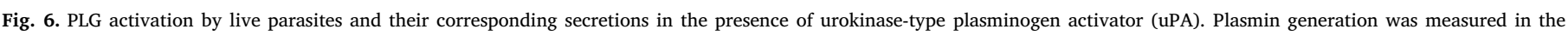

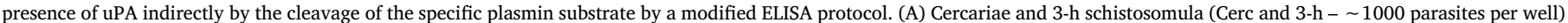

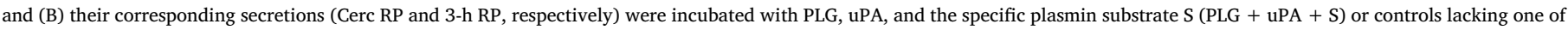

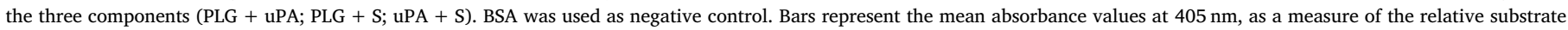

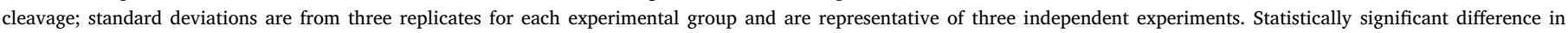
comparison to BSA is shown (*p $<0.05$, ** $\mathrm{p}<0.01$ ).

structure presenting a three-layer $\alpha-\beta-\alpha$ sandwich characteristic of the SCP/TAPS domain [26].

Similar to SmVAL4 protein [12], SmVAL18 was also identified in cercarial and 3-h schistosomula extracts and in their corresponding secretions (Cerc and 3-h RPs). These results are in agreement with data demonstrating that this protein is secreted during the first three hours of in vitro culturing $[5,10]$.

After the initial steps of cercarial adhesion to the human skin and entry into the epidermal layer, newly transformed schistosomula must overcome the tissue barriers of BM and ECM of the dermis in order to reach the venous circulation, where they must circumvent plasma components, e.g. complement proteins, en route to the lungs. We evaluated rSmVAL18 binding to ECM and plasma components using a modified ELISA protocol previously described for bacterial proteins $[18,29]$.

The recombinant protein was found to attach significantly to thrombin and plasminogen. Dissociation constant values $\left(K_{D}\right)$ of $62.5 \pm 20 \mathrm{nM}$ and $7.0 \pm 2.9 \mathrm{nM}$ were obtained for the binding of rSmVAL18 to thrombin and plasminogen (PLG), respectively. Although the recombinant protein showed specific attachment to thrombin, the formation of fibrin clots was not inhibited in vitro (data not shown).

The ability of rSmVAL18 to bind to PLG likely involves the Cterminal lysine residues of this recombinant protein, as we observed that 6-aminocaproic acid (ACA), a lysine analogue, was capable of almost totally inhibiting this interaction at $20 \mu \mathrm{M}$ concentration. In fact, our molecular modelling, based on the x-ray structure of SmVAL4 [16], shows that the recombinant protein has nine lysine residues at its molecular surface that could be interacting with PLG Kringle domains, a region known to frequently mediate interactions with lysine residues of bacterial receptors [30]. However, previous analysis of SCP/TAPS protein structures revealed that long flexible loops in each protein complicated the accurate prediction of their structures [16]. Thus, structural characterization is required in order to reveal the unique three-dimensional features of SmVAL18.

Plasminogen, abundant in human plasma and extracellular fluids, is converted into plasmin, its active form, by eukaryotic activators, such as tissue (tPA) and urokinase-type plasminogen activators (uPA). In mammals, plasmin is involved in the degradation of ECM proteins, blood clot dissociation (fibrinolysis) and cellular migration. While PLG is weakly activated in solution, this becomes dramatically enhanced through binding to plasminogen receptors [31,32]. The immobilization of plasminogen onto lysine-containing surfaces is associated with a dramatic conformational change in the molecule, making it more susceptible to TPA or uPA-mediated activation and more resistant to physiological inhibitors. As a result, the activation mechanism generates targeted, localized, and transient proteolytic activity [31].

Plasminogen-binding receptors were first identified as enolases in the tegument of Schistosoma bovis adult male worms (SbEno) and Schistosoma japonicum schistosomula (SjENO) [33-36]. Enolases were also identified in the secretions of adult worms of the trematodes Fasciola hepatica [37], Echinostoma caproni [38] and Clonorchis sinensis [39]. The recent characterization of the ability of live $S$. mansoni 7-day schistosomula and adult worms to enhance the activation of PLG was associated with a tegumental enolase (SmEno) [25]. Herein, we report the ability of live cercariae and 3-h schistosomula, as well as their corresponding secreted proteins, to increase the conversion of PLG into plasmin in vitro in the presence of uPA.

Many SmVAL family members have been identified as excreted/ secreted proteins during the infection of both the snail $[13,40]$ and the mammalian host $[5,10,12,15,26]$. In addition, gene transcripts were localized in specific tissues of larval and adult stages [12,14]. SmVAL9 stimulates the transcription of genes responsible for ECM degradation that likely create an environment suitable for miracidia to invade and sporocysts to migrate through Biomphalaria tissues [13,40]. Similarly, since SmVAL4 binds lipids, such as cholesterol [16], it probably acts in the lipid-signaling process to evade host immune responses, thus facilitating skin infection by cercariae. Its transcript was recently localized in pre-acetabular glands of germballs, along with SmVAL24 [12], SmVAL1, 10, 18 and 19 (Farias, personal communication). The synthesis of gland contents occurs during cercarial development (germballs), which enables the production of molecules to promote rapid host entry upon parasite emergence from the snail [5]. In adult worms, SmVAL6 transcript was observed in the oral/ventral suckers and in tegument cell bodies. SmVAL13 and 7 are probably associated with the blood feeding processes, since these were identified in the anterior and posterior esophageal glands, respectively [12,14]. Most of the relevant data presented above are summarized in Fig. 7. Based on extensive distribution throughout the different tissues in which SmVAL members have been identified, the notion of a unifying function for SmVALs seems unlikely, making it reasonable that the different members of this family may present distinct functions.

The ability of SmVAL18 to bind plasminogen, acting as a PLG-receptor, appears to be a mechanism that increases the local proteolytic activity of plasmin, allowing newly transformed schistosomula to reach blood vessels through the degradation of ECM components of the dermis. Taking into account previous findings and the data described herein, it would be reasonable to propose that the SmVAL proteins secreted during $S$. mansoni larval stages could act to overcome tissue barriers (SmVAL9 and SmVAL18) and the host immune mechanisms (SmVAL4) in order to facilitate the infection of both snails and 


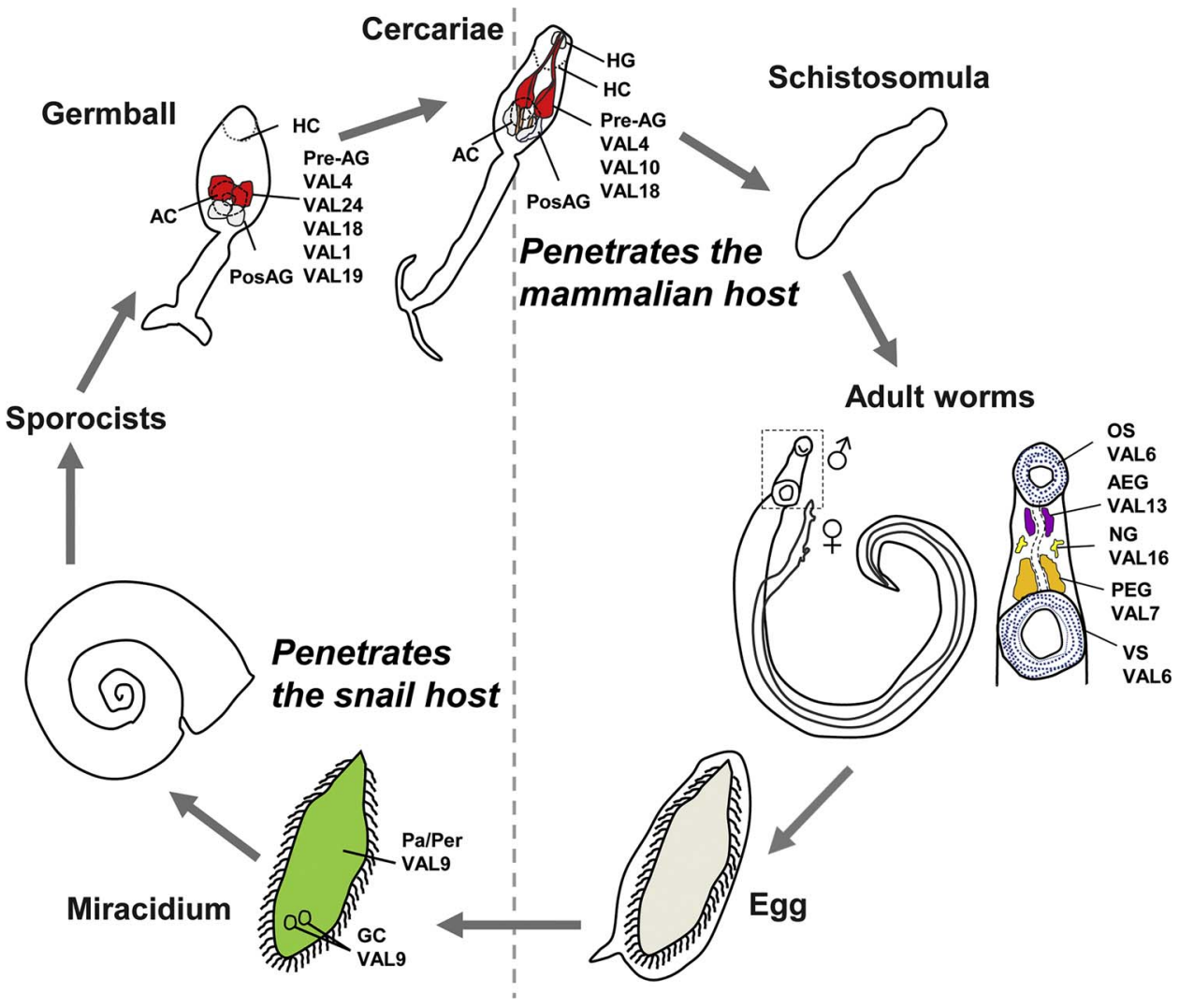

Fig. 7. SmVAL proteins/transcripts identified in specific tissues/structures of the intra-snail and intramammalian stages of the Schistosoma mansoni. SmVAL1, 4, 10, 18, 19 and 24 transcripts (in red) localized in the pre-acetabular glands of germballs at the final stage of development ([12] and Farias, personal communication); HC - Head Capsule, PreAG - Pre-acetabular glands, PosAG - Pos-acetabular glands, AC - Acetabulum. SmVAL4, 10 and 18 proteins (red) identified in the content of the pre-acetabular glands of cercariae [5,10,26]; HG - Head Gland. SmVAL6 (blue), 13 (purple), 16 (yellow) and 7 (orange) transcripts localized in the anterior region structures of male adult worms [12,14]; OS - Oral sucker, AEG - Anterior esophageal gland, NG Neural ganglia, PEG - Posterior esophageal gland, VS - Ventral Sucker. SmVAL9 protein (green) diffusely distributed in the parenchyma, perikarya and germinal cells of the miracidium [13]; Pa - Parenchyma, Per - perikarya, GC - Germinal Cells. Not to scale. (For interpretation of the references to colour in this figure legend, the reader is referred to the web version of this article.) mammalian hosts.

\section{Conclusions}

In this work we report that SmVAL18 is a plasminogen-binding protein secreted by cercariae and 3-h schistosomula. Interaction between SmVAL18 and PLG in the presence of a plasminogen activator (uPA) enhances PLG-plasmin conversion, which may promote local proteolytic activity that degrades the extracellular matrix of the dermis, thereby allowing parasites to reach the circulation.

\section{Acknowledgments}

We thank Andris Walter for English revision (Programa de Capacitação Fiotec) and Dr. Iain Chalmers (Aberystwyth University, Wales) for the kind gift of smVAL18 cDNA. This work was supported by Fundação Butantan and Fundação de Amparo à Pesquisa do Estado de São Paulo (FAPESP - 2012/23124-4 and FAPESP - 2010/18486-9).

\section{References}

[1] D.J. Gray, D.P. McManus, Y. Li, G.M. Williams, R. Bergquist, A.G. Ross, Schistosomiasis elimination: lessons from the past guide the future, Lancet Infect. Dis. 10 (10) (2010) 733-736.

[2] D.G. Colley, A.L. Bustinduy, W.E. Secor, C.H. King, Human schistosomiasis, Lancet 383 (9936) (2014) 2253-2264.

[3] B. Gryseels, K. Polman, J. Clerinx, L. Kestens, Human schistosomiasis, Lancet 368 (9541) (2006) 1106-1118.

[4] G.M. Knudsen, K.F. Medzihradszky, K.C. Lim, E. Hansell, J.H. McKerrow, Proteomic analysis of Schistosoma mansoni cercarial secretions, Mol. Cell. Proteom. 4 (12) (2005) 1862-1875.

[5] R.S. Curwen, P.D. Ashton, S. Sundaralingam, R.A. Wilson, Identification of novel proteases and immunomodulators in the secretions of schistosome cercariae that facilitate host entry, Mol. Cell. Proteom. 5 (5) (2006) 835-844.

[6] J.H. McKerrow, P. Jones, H. Sage, S. Pino-Heiss, Proteinases from invasive larvae of the trematode parasite Schistosoma mansoni degrade connective-tissue and basement-membrane macromolecules, Biochem. J. 231 (1) (1985) 47-51.

[7] C.H. Dorsey, C.E. Cousin, F.A. Lewis, M.A. Stirewalt, Ultrastructure of the Schistosoma mansoni cercaria, Micron 33 (3) (2002) 279-323.
[8] C.H. Dorsey, M.A. Stirewalt, Schistosoma mansoni: fine structure of cercarial acetabular glands, Exp. Parasitol. 30 (2) (1971) 199-214.

[9] A.J.M. Iain, Iain W. Chalmers, Richard M.R. Coulson, Marissa A. Wagner, Ralf Schmid, Hirohisa Hirai, Karl F. Hoffmann, Developmentally regulated expression, alternative splicing and distinct sub-groupings in members of the Schistosoma mansoni venom allergen-like (SmVAL) gene family, BMC Genom. 9 (2008) 89.

[10] J. Jang-Lee, R.S. Curwen, P.D. Ashton, B. Tissot, W. Mathieson, M. Panico, A. Dell, R.A. Wilson, S.M. Haslam, Glycomics analysis of Schistosoma mansoni egg and cercarial secretions, Mol. Cell. Proteom. 6 (9) (2007) 1485-1499.

[11] S. Parker-Manuel, Patterns of Gene Expression in Schistosoma Mansoni Larvae Associated with Infection of the Mammalian Host, Department of Biology, University of York, York, 2010.

[12] R.S. Fernandes, T.C. Barbosa, M.M.F. Barbosa, P.A. Miyasato, E. Nakano, L.C.C. Leite, L.P. Farias, Stage and tissue expression patterns of Schistosoma mansoni venom allergen-like proteins SmVAL 4, 13, 16 and 24, Parasite Vectors 10 (1) (2017) 223.

[13] T.P. Yoshino, M. Brown, X.J. Wu, C.J. Jackson, R. Ocadiz-Ruiz, I.W. Chalmers, M. Kolb, C.H. Hokke, K.F. Hoffmann, Excreted/secreted Schistosoma mansoni venom allergen-like 9 (SmVAL9) modulates host extracellular matrix remodelling gene expression, Int. J. Parasitol. 44 (8) (2014) 551-563.

[14] H.K. Rofatto, S.J. Parker-Manuel, T.C. Barbosa, C.A. Tararam, R. Alan Wilson, L.C. Leite, L.P. Farias, Tissue expression patterns of schistosoma mansoni venom allergen-like proteins 6 and 7, Int. J. Parasitol. 42 (7) (2012) 613-620.

[15] E. Hansell, S. Braschi, K.F. Medzihradszky, M. Sajid, M. Debnath, J. Ingram, K.C. Lim, J.H. McKerrow, Proteomic analysis of skin invasion by blood fluke larvae, PLoS Negl. Trop. Dis. 2 (7) (2008) e262.

[16] A. Kelleher, R. Darwiche, W.C. Rezende, L.P. Farias, L.C. Leite, R. Schneiter, O.A. Asojo, Schistosoma mansoni venom allergen-like protein 4 (SmVAL4) is a novel lipid-binding SCP/TAPS protein that lacks the prototypical CAP motifs, Acta Crystallogr. D: Biol. Crystallogr. (Pt. 8) (2014) 2186-2196.

[17] C.R. Ramos, P.A. Abreu, A.L. Nascimento, P.L. Ho, A high-copy T7 Escherichia coli expression vector for the production of recombinant proteins with a minimal Nterminal His-tagged fusion peptide, Braz. J. Med. Biol. Res. 37 (8) (2004) 1103-1109.

[18] A.S. Barbosa, P.A. Abreu, F.O. Neves, M.V. Atzingen, M.M. Watanabe, M.L. Vieira, Z.M. Morais, S.A. Vasconcellos, A.L. Nascimento, A newly identified leptospiral adhesin mediates attachment to laminin, Infect. Immun. 74 (11) (2006) 6356-6364.

[19] Y.P. Lin, D.W. Lee, S.P. McDonough, L.K. Nicholson, Y. Sharma, Y.F. Chang, Repeated domains of leptospira immunoglobulin-like proteins interact with elastin and tropoelastin, J. Biol. Chem. 284 (29) (2009) 19380-19391.

[20] M.L. Vieira, S.A. Vasconcellos, A.P. Goncales, Z.M. de Morais A.L. Nascimento, Plasminogen acquisition and activation at the surface of leptospira species lead to fibronectin degradation, Infect. Immun. 77 (9) (2009) 4092-4101.

[21] N. Eswar, B. Webb, M.A. Marti-Renom, M.S. Madhusudhan, D. Eramian, M.Y. Shen, 
U. Pieper, A. Sali, Comparative protein structure modeling using MODELLER, Curr. Protoc. Protein Sci. Chapter 2 (2007) Unit 2.9.

[22] J. Soding, A. Biegert, A.N. Lupas, The HHpred interactive server for protein homology detection and structure prediction, Nucleic Acids Res. 3 (Web Server issue) (2005) W244-W248

[23] V.B. Chen, W.B. Arendall 3rd, J.J. Headd, D.A. Keedy, R.M. Immormino, G.J. Kapral, L.W. Murray, J.S. Richardson, D.C. Richardson, MolProbity: all-atom structure validation for macromolecular crystallography, Acta Crystallogr. D: Biol Crystallogr. 66 (Pt. 1) (2010) 12-21.

[24] B.C. Figueiredo, A.A. Da'dara, S.C. Oliveira, P.J. Skelly, Schistosomes enhance plasminogen activation: the role of tegumental enolase, PLoS Pathog. 11 (12) (2015) e1005335.

[25] N.J. Greenfield, Using circular dichroism spectra to estimate protein secondary structure, Nat. Protoc. 1 (6) (2006) 2876-2890.

[26] L.P. Farias, D. Rodrigues, V. Cunna, H.K. Rofatto, E.L. Faquim-Mauro, L.C. Leite, Schistosoma mansoni venom allergen like proteins present differential allergic responses in a murine model of airway inflammation, PLoS Negl. Trop. Dis. 6 (2) (2012) e1510.

[27] A. Kelleher, R. Darwiche, W.C. Rezende, L.P. Farias, L.C.C. Leite, R. Schneiter, O.A. Asojo, I.U. Cr, Schistosoma mansoni venom allergen-like protein 4 (SmVAL4) is a novel lipid-binding SCP/TAPS protein that lacks the prototypical CAP motifs, Acta Crystallogr. D: Biol. Crystallogr. 70 (8) (2014) 2186-2196.

[28] S.J. Parker-Manuel, A.C. Ivens, G.P. Dillon, R.A. Wilson, Gene expression patterns in larval Schistosoma mansoni associated with infection of the mammalian host, PLoS Negl. Trop. Dis. 5 (8) (2011) e1274.

[29] L.G.V. Fernandes, M.L. Vieira, K. Kirchgatter, I.J. Alves, Z.M. de Morais, S.A. Vasconcellos, E.C. Romero, A. Nascimento, OmpL1 is an extracellular matrixand plasminogen-interacting protein of Leptospira spp, Infect. Immun. (2012) 3679-3692.

[30] K. Lahteenmaki, P. Kuusela, T.K. Korhonen, Bacterial plasminogen activators and receptors, FEMS Microbiol. Rev. 25 (5) (2001) 531-552.

[31] K. Lahteenmaki, R. Virkola, R. Pouttu, P. Kuusela, M. Kukkonen, T.K. Korhonen, Bacterial plasminogen receptors: in vitro evidence for a role in degradation of the mammalian extracellular matrix, Infect. Immun. 63 (9) (1995) 3659-3664.

[32] A.Y. Jong, S.H. Chen, M.F. Stins, K.S. Kim, T.L. Tuan, S.H. Huang, Binding of Candida albicans enolase to plasmin(ogen) results in enhanced invasion of human brain microvascular endothelial cells, J. Med. Microbiol. 2 (Pt. 8) (2003) 615-622.

[33] A. Ramajo-Hernandez, R. Perez-Sanchez, V. Ramajo-Martin, A. Oleaga, Schistosoma bovis: plasminogen binding in adults and the identification of plasminogen-binding proteins from the worm tegument, Exp. Parasitol. 115 (1) (2007) 83-91.

[34] E. de la Torre-Escudero, R. Manzano-Roman, R. Perez-Sanchez, M. Siles-Lucas, A. Oleaga, Cloning and characterization of a plasminogen-binding surface-associated enolase from Schistosoma bovis, Vet. Parasitol. 173 (1-2) (2010) 76-84.

[35] J. Yang, C. Qiu, Y. Xia, L. Yao, Z. Fu, C. Yuan, X. Feng, J. Lin, Molecular cloning and functional characterization of Schistosoma japonicum enolase which is highly expressed at the schistosomulum stage, Parasitol. Res. 107 (3) (2010) 667-677.

[36] Y. Cai, R. Wang, B. Liang, N. Bai, Y. Liu, Systematic review and meta-analysis of the effectiveness and safety of tigecycline for treatment of infectious disease, Antimicrob. Agents Chemother. 55 (3) (2011) 1162-1172.

[37] D. Bernal, J.E. de la Rubia, A.M. Carrasco-Abad, R. Toledo, S. Mas-Coma, A. Marcilla, Identification of enolase as a plasminogen-binding protein in excretorysecretory products of Fasciola hepatica, FEBS Lett. 563 (1-3) (2004) 203-206.

[38] A. Marcilla, A. Perez-Garcia, A. Espert, D. Bernal, C. Munoz-Antoli, J.G. Esteban, R. Toledo, Echinostoma caproni: identification of enolase in excretory/secretory products molecular cloning, and functional expression, Exp. Parasitol. 117 (1) (2007) 57-64.

[39] X. Wang, W. Chen, F. Hu, C. Deng, C. Zhou, X. Lv, Y. Fan, J. Men, Y. Huang, J. Sun, D. Hu, J. Chen, Y. Yang, C. Liang, H. Zheng, X. Hu, J. Xu, Z. Wu, X. Yu, Clonorchis sinensis enolase: identification and biochemical characterization of a glycolytic enzyme from excretory/secretory products, Mol. Biochem. Parasitol. 177 (2) (2011) $135-142$.

[40] X.J. Wu, G. Sabat, J.F. Brown, M. Zhang, A. Taft, N. Peterson, A. Harms, T.P. Yoshino, Proteomic analysis of Schistosoma mansoni proteins released during in vitro miracidium-to-sporocyst transformation, Mol. Biochem. Parasitol. 164 (1) (2009) 32-44. 